\title{
Ultraviolet laser transverse profile shaping for improving $x$-ray free electron laser performance
}

\author{
S. Li, ${ }^{*}$ S. Alverson, D. Bohler, A. Egger, A. Fry, S. Gilevich, \\ Z. Huang, A. Miahnahri, D. Ratner, J. Robinson, and F. Zhou \\ SLAC National Accelerator Laboratory, 2575 Sand Hill Road, Menlo Park, California 94025, USA
}

(Received 3 June 2017; published 17 August 2017)

\begin{abstract}
The photocathode rf gun is one of the most critical components in $\mathrm{x}$-ray free electron lasers. The drive laser strikes the photocathode surface, which emits electrons with properties that depend on the shape of the drive laser. Most free electron lasers use photocathodes with work function in the ultraviolet, a wavelength where direct laser manipulation becomes challenging. In this paper, we present a novel application of a digital micromirror device (DMD) for the $253 \mathrm{~nm}$ drive laser at the Linear Coherent Light Source. Laser profile shaping is accomplished through an iterative algorithm that takes into account shaping error and efficiency. Next, we use laser shaping to control the X-ray laser output via an online optimizer, which shows improvement in FEL pulse energy. Lastly, as a preparation for electron beam shaping, we use the DMD to measure the photocathode quantum efficiency across cathode surface with an averaged laser rms spot size of $59 \mu \mathrm{m}$. Our experiments demonstrate promising outlook of using DMD to shape ultraviolet lasers for photocathode rf guns with various applications.
\end{abstract}

DOI: 10.1103/PhysRevAccelBeams.20.080704

\section{INTRODUCTION}

The x-ray free electron laser (FEL) is the fourth generation light source that produces high power, tunable, and coherent X-rays. Relativistic electrons traveling in alternating magnets, or undulators, radiate coherent $\mathrm{x}$-rays via resonant interaction with emitted photons [1]. Current state of the art FEL facilities utilize photocathode rf guns to produce high brightness electron beams [2-4]. The drive laser strikes the photocathode surface to emit electrons. The temporal and transverse shape of the laser pulse, together with the quantum efficiency (QE) of the photocathode surface, determines the temporal and transverse distribution of the electron beam upon emission, which has a significant impact on beam brightness and FEL performance [5-10]. The temporal distribution can be manipulated with techniques such as polarization based pulse stacking with birefringent crystals [11], acousto-optic modulators $[12,13]$ or spatial modulators at a dispersion region [14]. The transverse profile shaping is also complicated since it involves nonuniformities from the drive laser and the photocathode QE spatial variation. These structures can carry over to the electron beam and can degrade beam brightness and FEL performance. Past studies have shown that certain laser profiles lead to lower electron beam

\footnotetext{
*siqili@slac.stanford.edu
}

Published by the American Physical Society under the terms of the Creative Commons Attribution 4.0 International license. Further distribution of this work must maintain attribution to the author(s) and the published article's title, journal citation, and DOI. emittance [15-17]. It is of significant interest to be able to control the transverse profile of the drive laser, and to study its influence on electron beam properties.

At most x-ray FEL facilities, transverse shaping of the drive laser is done by expanding the beam before an iris to create a roughly cut-Gaussian or flat-top profile, not addressing the fine nonuniformities in the beam. Moreover, the cathode $\mathrm{QE}$ evolves over time due to extended exposure at specific locations. Therefore, laser transverse shaping calls for a more flexible and adaptive solution to address the detailed structures in the beam. Recent studies have used liquid crystal based spatial light modulators (SLMs) to achieve drive laser shaping for Cornell's high voltage dc gun at $532 \mathrm{~nm}[18,19]$ and for PITZ drive laser at $1030 \mathrm{~nm}$ [20]. The working mechanism of liquid crystal SLMs is through modulating the phase of the incident light by changing the refractive index in each liquid crystal pixel. Due to the nature of liquid crystals, these types of SLMs are designed to work properly and safely from visible light to IR wavelengths. Photocathode materials that reduce the work function to visible wavelengths, such as $\mathrm{Cs}_{3} \mathrm{Sb}, \mathrm{GaAs}$, and Alkali Antimonide, have been studied and developed in the community [21-23]. However, FEL facilities with high gradient, high frequency rf guns still utilize metal photocathodes for their robustness and sustainability under years of high fields to produce high brightness beams [24]. Metal photocathodes require UV photons due to their high work function, therefore eliminating the use of liquid crystal SLMs directly in this wavelength range. We resort to a different type of adaptive optics, digital micromirror device (DMD), that can work in UV with some hardware manipulation. The convenience of 
laser shaping at UV allows the possibility of inserting the device close to the photocathode, therefore reducing the fluence on the device to well below damage threshold, and potentially increasing the transport efficiency. Shaping the laser at UV wavelengths paves the way for further application in other XFEL facilities, or any UV photocathode electron sources.

In this paper, we present experimental methods and results of using DMD in the Linear Coherent Light Source (LCLS) beam line. We describe an iterative algorithm to achieve fine shaping in the transverse profile of the drive laser. Next, we will focus on two specific applications of the laser shaping technique. First, we show that by varying the drive laser transverse profile we can control the final x-ray pulse energy with an online FEL optimizer, Ocelot [25]. The possibility of flexible tuning of the drive laser to optimize FEL presents a great advantage over manually adjusting optics in the drive laser system that can cost an appreciable amount of time and human effort. We briefly discuss our preliminary emittance measurements while varying the drive laser profile. Second, we present the cathode QE measurement with DMD. Measuring QE across the cathode surface provides knowledge of the cathode performance over time, and helps with improving electron source qualities, which will be discussed below.

The structure of the paper is as follows. In Sec. II we describe the working mechanism of the DMD and give an overview of the optical properties related to the experiment. Next, we briefly discuss the algorithm to achieve laser shaping and present results of the LCLS drive laser beam. In Sec. IV, we demonstrate using DMD to optimize LCLS $\mathrm{x}$-ray pulse energy. Furthermore, we show the measurement of LCLS photocathode QE map using the DMD and discuss future work in electron beam shaping. Lastly, we discuss potential applications of laser shaping and further improvements to our experiments.

\section{EXPERIMENTAL SETUP}

There are many commercially available adaptive optics for the purpose of laser shaping. As mentioned above, J. Maxson et al. [19] have used liquid-crystal-on-silicon (LCoS) spatial light modulators (SLM) to spatially modulate the drive laser at $532 \mathrm{~nm}$ wavelength at Cornell. A. Halavanau et al. [26] have used a microlens array to experimentally characterize and shape the beam transverse profile. Other relevant studies can be found in [7,27]. At LCLS, a copper photocathode demands a UV drive laser at $253 \mathrm{~nm}$ [28]. After extensive damage tests on various materials [29], we choose to work with the digital micromirror device (DMD) from Texas Instruments [30]. Commercially available DMDs do not work in deep UV because the window on the chip only transmits wavelengths down to $363 \mathrm{~nm}$ [31]. We contacted a Maryland company, $\mathrm{L}-1$, to swap the window to $\mathrm{CaF} 2$ with $\mathrm{AR}$ coating. Damage tests show that a converted UV DMD can sustain

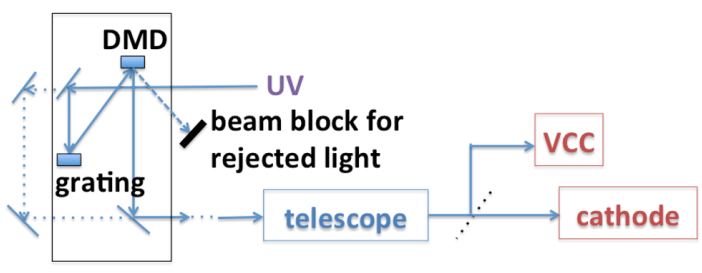

FIG. 1. Simplified layout of the LCLS injector laser imaging system with the DMD and grating installed.

up to $90 \mu \mathrm{J}$ laser pulse energy in a $1 \mathrm{~cm}$ diameter spot, with a peak fluence of $0.51 \mathrm{~mJ} / \mathrm{cm}^{2}$, when the 2 ps full-width half-maximum laser pulse is timed to arrive after the micromirrors have just stabilized into a new state for each period (see details in [30]). Note that the maximum acceptable fluence depends on the integrated pulse energy.

The model we use is the Texas Instruments DLP7000 DMD which consists of $768 \times 1024$ micromirrors with size $13.68 \mu \mathrm{m}$. The micromirrors can flip into two states, ON or OFF corresponding to $+/-12^{\circ}$, given an input voltage. Due to the geometry of the DMD mirrors, it is only possible to reduce rather than modulate the laser intensity. To reduce the laser intensity by a certain amount, we group the individual micromirrors into macropixels and turn off a fraction of randomly-distributed micromirrors in each macropixel. The device is programmable through an application program interface in Matlab, which we incorporate into the LCLS control system.

The optical layout of the system is determined by the geometrical design of the DMD mirrors. The structure of the micromirrors introduces a pulse front tilt as from a grating. The pulse front tilt is effectively a correlation between the time coordinate and the transverse coordinate. For example, a laser beam with diameter of $1 \mathrm{~cm}$ with normal incident on the DMD results in a pulse front tilt of $13.6 \mathrm{ps}$, which is significant for a 2 ps laser pulse. For a photocathode rf gun, this effect leads to an elongated electron bunch length. We compensate this effect by introducing a diffraction grating upstream of the DMD, which cancels the pulse front tilt from the DMD. The compensation is confirmed by measuring the electron bunch length in comparison to the regular setup without DMD, which shows less than $10 \%$ difference and is within measurement uncertainty. The grating-DMD system is installed in the LCLS injector laser beam line with easy transition from and back to the regular setup without it. When installed, the DMD plane is imaged to the photocathode. We utilize the virtual cathode camera (VCC) as our target plane for shaping (Fig. 1).

\section{LASER SHAPING}

\section{A. Mapping the DMD plane and the target plane}

In order to achieve accurate shaping we need to have a mapping relation between the DMD plane and the target 


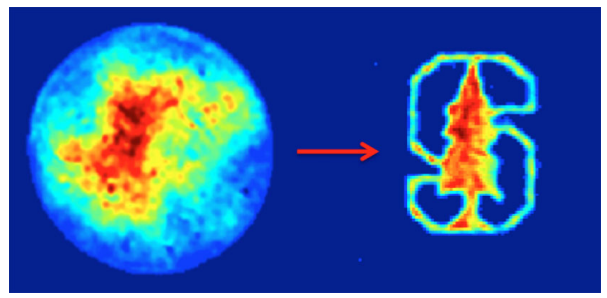

FIG. 2. Original beam profile (left) and Stanford tree profile at VCC (right).

(VCC) plane. We assume a linear transformation between the two planes, which takes into account magnification, rotation, skewing, and mirror imaging. We follow the procedures described in [19]. It is important to note that this method can be used in any target plane as long as the imaging process is linear. As a demonstration, we shape the beam into a Stanford tree on VCC to show the correct mapping relation, Fig. 2.

\section{B. Shaping the LCLS drive laser}

We implement an algorithm that iteratively shapes the laser. The $768 \times 1024$ micromirrors are grouped into $5 \times 5$ macropixels. Turning off a fraction of randomly-distributed micromirrors in each macropixel reduces intensity to a desired value. The user initially provides a general choice of target shape, such as flat-top, cut-Gaussian, or parabolic. Considering that the DMD only reduces intensity, the user also provides a minimum shaping efficiency requirement. The first iteration defines a cost function in terms of shaping error and shaping efficiency, Cost $=a \times$ Error + $b \times \operatorname{sigmoid}\left(E-E_{\text {calc }}\right)$ where Error refers to the shaping error, $E$ the minimum efficiency, $E_{\text {calc }}$ the calculated shaping efficiency, and $a$ and $b$ are found empirically (we usually set $b \gg a$ to guarantee the efficiency requirement). The sigmoid function ensures that when the

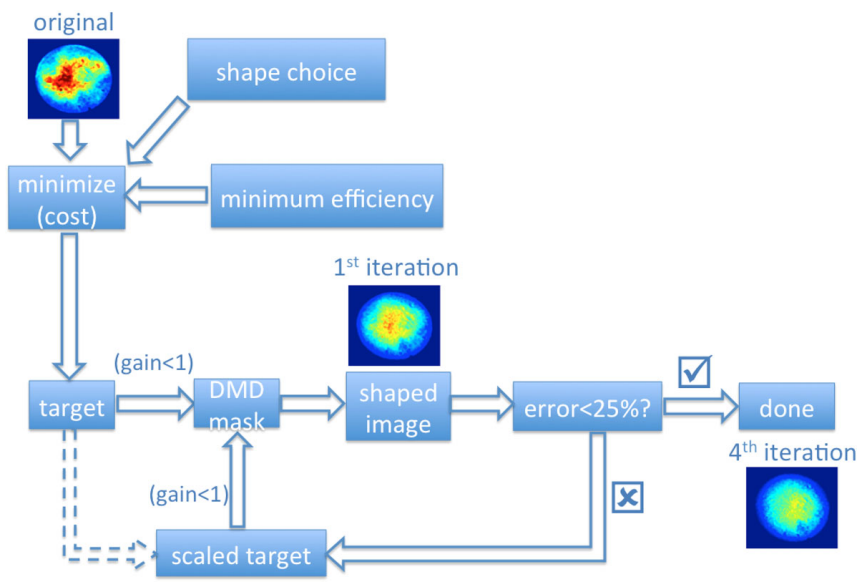

FIG. 3. Flow chart of the shaping algorithm, with example VCC images of the laser beam in the original shape, after the first iteration, and after the fourth iteration. calculated efficiency is below the minimum requirement, the cost function will blow up in order for the algorithm to reject this shape. By minimizing the cost function, we are able to find specific parameters for the target shape that meets the user requirements. Due to systematic errors in the optical transport we observe overshooting of the shaping target. Therefore the shaping gain is introduced as the ratio of the number of real micromirrors to turn off to the calculated number of micromirrors to turn off. To avoid overshooting the target, we set gain to be less than 1 and allow multiple iterations to gradually approach the ultimate target shape. Between iterations we rescale the target shape to gradually correct for the difference between the shaped profile and the ultimate target profile. The shaping algorithm is illustrated in the diagram Fig. 3. So far, we have implemented successful laser shaping for multiple types of profiles (Fig. 4).

The shaping resolution is determined by the micromirror grouping and the optical transport. In our case of a $5 \times 5$ grouping, the macropixel size corresponds to a square of size $68.4 \mu \mathrm{m}$. The optical transport at LCLS results in an approximately $12 \times 12 \mu \mathrm{m}$ square on the cathode, which is sufficient resolution to correct for typical hot spots in the drive laser (see for example, the original laser image in Fig. 4 upper left).


FIG. 4. Example VCC images of laser shaping. Top left is the original laser profile. Top right is cut-Gaussian shaping (error: 18\%, efficiency: $77 \%$ ). Bottom left is flat-top shaping (error: 23\%, efficiency: $71 \%$ ). Bottom right is parabolic shaping (error: 19\%, efficiency: 67\%). Note that the color scale for the shaped profiles (top right, lower left, and lower right) is $60 \%$ of the color scale of the original beam image (top left), in order to show stronger contrast. Since the DMD can only reduce intensity, the fine structures in the shaped profiles, for example the lower right corner of the flat-top image, come from the corresponding low intensity part of the original beam. This indicates that the shaping performance also depends on the uniformity of the initial beam. 


\section{FEL OPTIMIZATION}

At LCLS, the drive laser profile drifts overtime and moves away from the optimal condition for FEL performance, which requires operators to steer transport mirrors every few days. Every few weeks laser engineers hand-tune the laser system. Both of these operations are costly in terms of interrupting beam delivery and human capital. We apply the laser shaping technique to ease these operational difficulties and optimize the FEL performance.

A Bayesian optimizer, Ocelot, has been developed and used in production as an online optimizer [25]. Ocelot can handle arbitrary machine parameters and objective functions for maximization. In the case of laser shaping, we limit the scanning variables to those only relevant to the drive laser profile, while keeping all other machine parameters fixed. For this study, we focus on a cut-Gaussian type shaping. We use the following three parameters to characterize the drive laser profile: $x$ offset, $y$ offset, and the cut ratio. The $x$ and $y$ offsets describe the beam centroid offset relative to the aperture center in units of DMD pixels. The cut ratio is defined as the ratio of the intensity at the cut radius to the center intensity. According to [16], the optimal cut ratio is empirically found to be 0.5 , corresponding to a half-maximum truncation.
We conducted a scan of 39 iterations, targeting to optimize FEL pulse energy. The scan starts with an intentionally off-centered, widely cut beam profile, which represents a typical situation of laser drifting at LCLS. In the end, Ocelot converges to a solution with the beam centroid close to the aperture center and cuts around half maximum. Constant efficiency requirement ensures the charge fluctuation to be less than $10 \%$, centered around $110 \mathrm{pC}$. On the day of measurement the laser heater is not available which causes a drop in x-ray pulse energy compared to usual operation at LCLS. With an intentionally distorted drive laser profile, the $\mathrm{x}$-ray pulse energy is initially below $200 \mu \mathrm{J}$. Together with online drive laser shaping, the Ocelot scan improves the FEL gas detector reading by more than a factor of 2 . The evolution of the beam shape and scanning parameters are shown in Fig. 5 and Fig. 6 respectively. These plots clearly show a convergence towards an optimum where the beam centroid is close to aperture center ( $x$ and $y$ offset close to 0 ), and the beam is cut at half maximum (cut ratio close to 0.5 ).

Without extensive beam matching and injector tuning, our preliminary emittance measurement shows improvement in emittance as the drive laser profile changes. In the left panel of Fig. 7, we use the intentionally off-centered

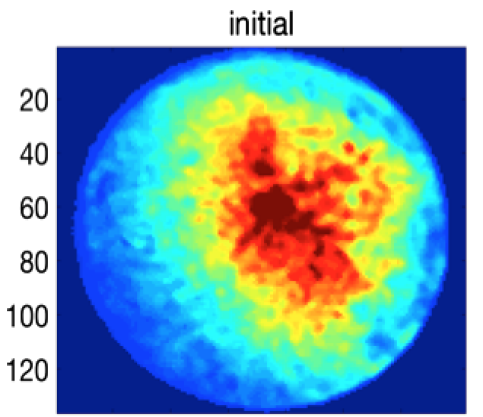

$20 \quad 40 \quad 60 \quad 80 \quad 100 \quad 120$

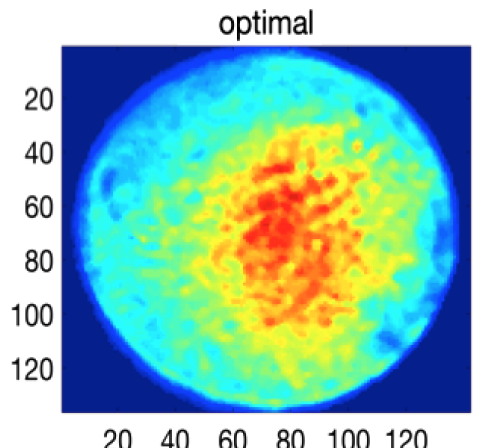

$\begin{array}{llllll}20 & 40 & 60 & 80 & 100 & 120\end{array}$

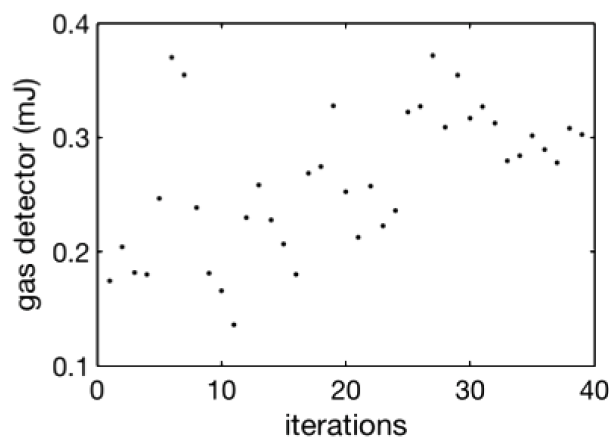

FIG. 5. Left: initial beam profile corresponding to $175 \mu \mathrm{J}$. Middle: optimal beam profile corresponding to $372 \mu \mathrm{J}$. Right: gas detector reading evolution through 39 iterations.
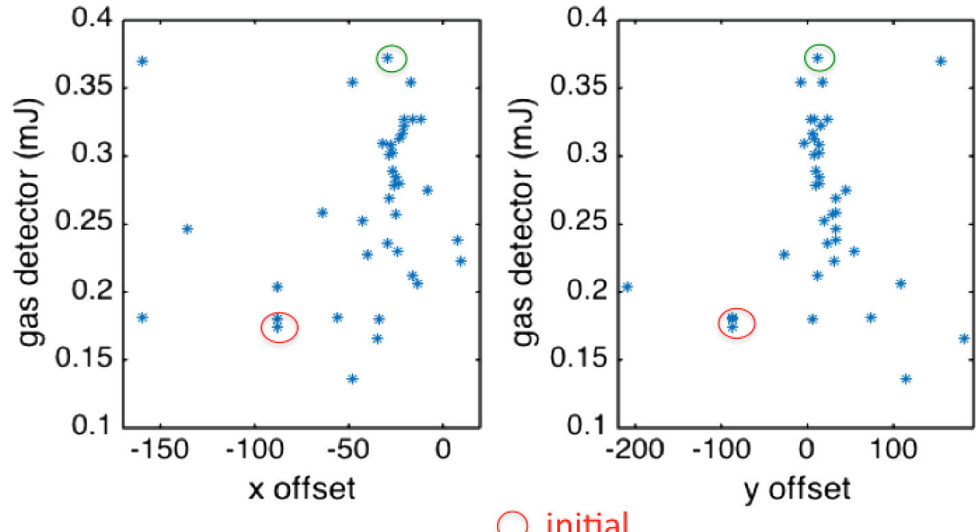

initial

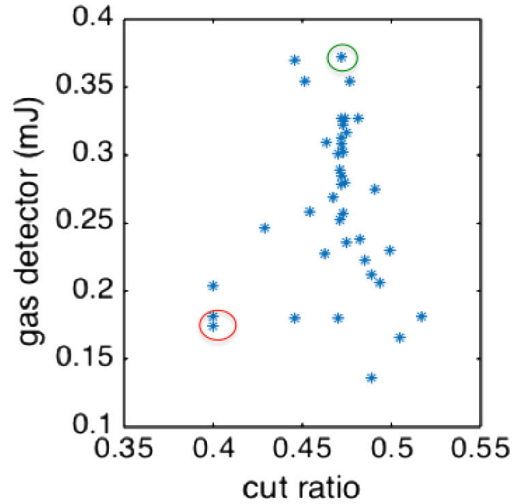

optimal

FIG. 6. The evolution of the FEL gas detector reading as a function of each of the variables. 

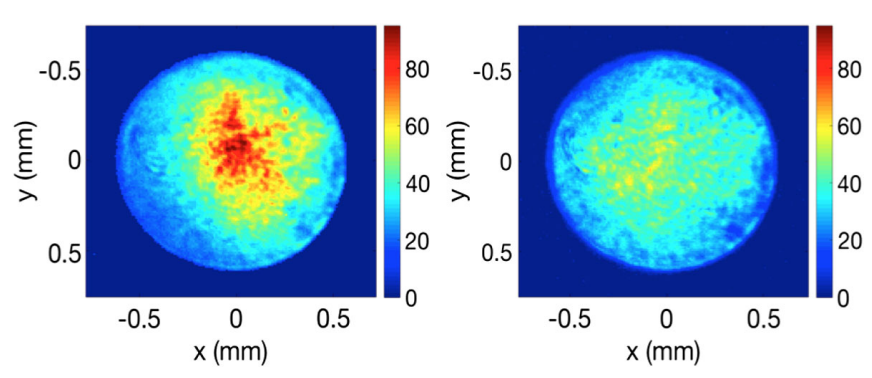

FIG. 7. Drive laser profiles for preliminary emittance measurements.

profile, which is the initial profile in the Ocelot scan described above. This drive laser profile gives an emittance of $0.56 \mu \mathrm{m}$ in $x$ and $0.53 \mu \mathrm{m}$ in $y$. In the right panel of Fig. 7, we use the centered, cut at half-maximum, truncated-Gaussian profile. This profile gives an emittance of $0.47 \mu \mathrm{m}$ in $x$ and $0.41 \mu \mathrm{m}$ in $y$. This result is consistent with previous studies on the effect of the drive laser profile on the electron beam emittance $[16,15]$. However, we emphasize that these measurements are preliminary, and we do not draw any conclusions solely based on these measurements. Beam emittance depends on a number of important factors other than the drive laser transverse profile, such as photocathode QE, beam matching, and the measurement method, which are not rigorously controlled or addressed here. We consider electron profile shaping as the immediate next step toward using the laser shaping technique to improve the electron beam emittance. With this tool in hand, one can conduct insightful studies on the effects of electron beam profile on beam brightness and use this knowledge to control the FEL performance. The prospects of achieving electron beam shaping will be discussed in detail below.

\section{PHOTOCATHODE QUANTUM EFFICIENCY SCAN}

Traditionally the photocathode QE has been measured by steering a small laser spot (usually on the order of tens of microns) across the cathode [32,33]. Without automatic control of the steering, this process requires careful monitoring and stabilization of the laser power, steering mirrors, and various machine parameters. Spatial light modulators, such as DMD, become the perfect candidate for this purpose. In order to obtain a $\mathrm{QE}$ profile, we need to know the charge counts corresponding to a localized laser illumination on the photocathode. We capture the electron emission on a YAG screen downstream of the cathode. To avoid saturation we lower laser power to reduce charge to $10 \mathrm{pC}$ level. The total count of the signal is a measure of charge. We turn on a small square of pixels on the DMD and move it across the laser profile. For each square, we obtain the charge counts, laser intensity, and laser spot location from the YAG image and VCC image. The

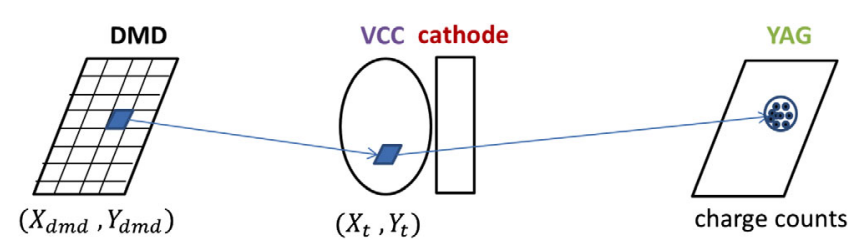

FIG. 8. Illustration of raster scan to obtain a QE map across the laser illumination area on cathode.

procedure of the raster scan is illustrated in Fig. 8. The mapping between the DMD plane and the laser camera plane is also obtained during the scan. To avoid measurement and analysis bias which leads to a correlation between measured QE and laser intensity, each time before we start a raster scan, we shape the laser profile into a flat-top beam on VCC, and then apply the flat-top mask together with the square to raster scan the cathode. In this way, we ensure that the charge counts on YAG reflect the QE of the cathode. To obtain the absolute $\mathrm{QE}$ in photons/electron, we calibrate the laser intensity to laser camera intensity with a full laser beam image, and likewise for the electron counts. We move the laser around the cathode to expand the region of the raster scan and merge the results together. Figure 9 shows the results from two scans with calibrated QE. Comparing the overlapping region of two independent scans, we find $10 \%$ errors on the measurements. The measurement indicates a large variation of $\mathrm{QE}$ over the scanned surface. The potential of using the DMD to compensate for the $\mathrm{QE}$ nonuniformities becomes favorable for electron beam shaping, as will be discussed below.

The measurement resolution is determined by the DMD pixel size, magnification/demagnification factor in the optical beam line, and the signal to noise ratio of the laser signal and electron signal on corresponding screens. The highest resolution we can achieve is $30 \times 30 \mathrm{DMD}$ pixels, taking into account of optical demagnification, which gives

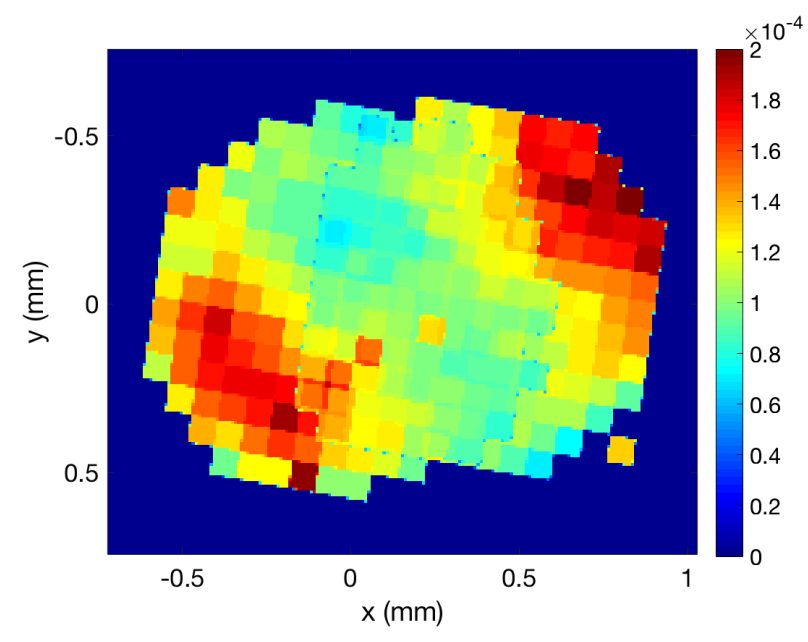

FIG. 9. QE map from combination of two partially overlapping raster scans. Blue region is not scanned by the laser. 
an averaged laser spot size of $59 \mu \mathrm{m}$ on the cathode. In terms of measurement time, the DMD can change pattern every $44 \mu \mathrm{s}$, faster than the LCLS repetition rate. This means that in principle the measurement can be done in minutes, ignoring machine conditions such as safety requirement to reduce repetition rate. This feature is favorable for operation because one can easily monitor the cathode quality over time.

Using spatial modulators such as DMD to measure QE across the photocathode has many potential applications. Specifically, this measurement prepares us for electron beam shaping. Compared to shaping the laser, the complication of shaping the electron beam comes in from mapping the target plane to the DMD plane. The electron imaging from cathode to a downstream image plane is not linear as in the optical case. For example, the magnification depends on the charge, which again depends on the QE of the cathode. Therefore, instead of following the mapping procedure as in the laser shaping case, we can choose the target plane immediately after the cathode, where the electron emission profile is the convolution of the laser profile and the QE profile of the cathode. Knowing the localized QE across the cathode surface, we can feed the measured electron emission profile into the shaping algorithm in Sec. III B. In this way, we can produce arbitrary electron shapes. With proper machine settings to perfectly image the electron beam at low current while avoiding space charge effects, the electron beam profile can be preserved along the accelerator. In addition, knowing the QE map across the photocathode surface, we can move the laser beam toward the higher QE region to deliver higher electron charge if needed, as well as reducing power loss in the shaping process.

\section{CONCLUSIONS AND OUTLOOK}

In this paper, we show that we can use a digital micromirror device to produce arbitrary laser profiles at the LCLS injector. We describe two specific applications of drive laser shaping-FEL optimization and photocathode QE measurement. We optimize the $\mathrm{x}$-ray laser output by varying the drive laser profile through an online optimizer while keeping all other knobs fixed. The increase in FEL pulse energy shows that indeed laser shaping provides appreciable improvement and convenience in operation. Instead of using the beam centroid location and cut Gaussian width, one can choose more parameters to characterize the drive laser shape. Ideally, the states of the micromirrors can be interpreted as parameters for the optimization process, exploiting the flexibility and high resolution features of the DMD. The photocathode QE measurement using the DMD demonstrates a simple and fast procedure that provides us with an easy access to the photocathode condition for daily operation. The most direct benefit of QE measurement is to prepare for electron beam shaping. Given a detailed QE map, the electron emission can be shaped into any arbitrary profiles using the same shaping algorithm as in the drive laser shaping case. This opens up enormous potential for studying electron beam accelerators. As mentioned above, one can study the effect of transverse beam profile on emittance and eventually control the beam emittance to manipulate FEL power. In addition, one can also put various patterns on the beam to measure standard optical parameters of the injector, such as the point spread function and the optical transfer function [34].

Our experiment can be improved in several ways. As described in the caption of Fig. 4, the accuracy of shaping depends on the uniformity of the initial beam shape. Reference [26] demonstrates a flat-top laser shaping technique using microlens arrays. This type of optics can be inserted in the beam line upstream of the adaptive optics section to create a more uniform initial beam. In this way, we can avoid low intensity holes in the initial laser that leads to imperfect shaping. Moreover, our experiment is limited by the damage threshold of the DMD, which can produce 100 to $150 \mathrm{pC}$ in our system. Even though many experiments can run at this charge level, it is more reliable and convenient if we can safely handle $250 \mathrm{pC}$ without running the risk of damaging the DMD. One solution is to place the DMD further downstream and closer to the cathode if space allows. Another solution is to use a customized DMD in UV that handles more laser power. The latter is currently under investigation.

In light of the promising results of drive laser shaping in rf photocathode gun shown in this paper, our study paves the way for future application of laser shaping to free electron laser facilities and any UV photocathode electron sources.

\section{ACKNOWLEDGMENTS}

We would like to thank D. Dowell, B. Dunham, R. Fiorito, J. Maxson, E. Montgomery for helpful discussions and suggestions. Work is supported by Department of Energy Contract No. DE-AC02-76SF00515.

[1] Z. Huang and K.-J. Kim, Review of x-ray free-electron laser theory, Phys. Rev. ST Accel. Beams 10, 034801 (2007).

[2] J. Fraser, R. Sheffield, and E. Gray, A new high-brightness electron injector for free electron lasers driven by RF linacs, Nucl. Instrum. Methods Phys. Res., Sect. A 250, 71 (1986).

[3] L. Serafini and J. B. Rosenzweig, Envelope analysis of intense relativistic quasilaminar beams in rf photoinjectors: mA theory of emittance compensation, Phys. Rev. E 55, 7565 (1997).

[4] D. H. Dowell, Sources of Emittance in RF Photocathode Injectors: Intrinsic emittance, space charge forces due to 
non-uniformities, RF and solenoid effects, arXiv:1610.01242.

[5] O. J. Luiten, S. B. vanderGeer, M. J. deLoos, F. B. Kiewiet, and M. J. vanderWiel, How to Realize Uniform ThreeDimensional Ellipsoidal Electron Bunches, Phys. Rev. Lett. 93, 094802 (2004).

[6] J. Rosenzweig, A. Cook, R. England, M. Dunning, S. Anderson, and M. Ferrario, Emittance compensation with dynamically optimized photoelectron beam profiles, Nucl. Instrum. Methods Phys. Res., Sect. A 557, 87 (2006).

[7] P. Musumeci, J. T. Moody, R. J. England, J. B. Rosenzweig, and T. Tran, Experimental Generation and Characterization of Uniformly Filled Ellipsoidal Electron-Beam Distributions, Phys. Rev. Lett. 100, 244801 (2008).

[8] I. V. Bazarov, B. M. Dunham, and C. K. Sinclair, Maximum Achievable Beam Brightness from Photoinjector, Phys. Rev. Lett. 102, 104801 (2009).

[9] Y. Li, S. Chemerisov, J. Lewellen, Laser pulse shaping for generating uniform three-dimensional ellipsoidal electron beams, Phys. Rev. ST Accel. Beams 12, 020702 (2009).

[10] C. Emma, J. Wu, K. Fang, S. Chen, S. Serkez, and C. Pellegrini, Terawatt X-ray free-electron-laser optimization by transverse electron distribution shaping, Phys. Rev. ST Accel. Beams 17, 110701 (2014).

[11] I. V. Bazarov, D. G. Ouzounov, B. M. Dunham, S. A. Belomestnykh, Y. Li, X. Liu, R. E. Meller, J. Sikora, C. K. Sinclair, F. W. Wise et al., Efficient temporal shaping of electron distributions for high-brightness photoemission electron guns, Phys. Rev. ST Accel. Beams 11, 040702 (2008).

[12] C. W. Hillegas, J. X. Tull, D. Goswami, D. Srickland, and W. S. Warren, Femtosecond laser pulse shaping by use of microsecond radio-frequency pulses, Opt. Lett. 19, 737 (1994).

[13] A. Marinelli, R. Coffee, S. Vetter, P. Hering, G. N. West, S. Gilevich, A. A. Lutman, S. Li, T. Maxwell, J. Galayda, A. Fry, and Z. Huang, Optical Shaping of X-Ray FreeElectron Lasers, Phys. Rev. Lett. 116, 254801 (2016).

[14] J. Yang, F. Sakai, T. Yanagida, M. Yorozu, Y. Okada, K. Takasago, A. Endo, A. Yada, and M. Washio, Lowemittance electron-beam generation with laser pulse shaping in photocathode radio-frequency gun, J. Appl. Phys. 92, 1608 (2002).

[15] A. Brachmann, R. N. Coffee, D. H. Dowell, S. A. Edstrom, P. Emma, S. Gilevich, G. Hays, P. Hering, Z. Huang, A. Miahnahri, H.-D. Nuhn, D. Ratner, D. A. Reis, W. White, J. Wu, and D. Xiang, LCLS Drive Laser Shaping Experiments, in Proceedings of FEL'2009, Liverpool, UK, 2009 (STFC Daresbury Laboratory, Warrington, 2009), p. 463.

[16] F. Zhou, A. Brachmann, P. Emma, S. Gilevich, and Z. Huang, Impact of the spatial laser distribution on photocathode gun operation, Phys. Rev. ST Accel. Beams 15, 090701 (2012).

[17] C. Gulliford, A. Bartnik, I. Bazarov, B. Dunham, and L. Cultrera, Demonstration of cathode emittance dominated high bunch charge beams in a DC gun-based photoinjector, Appl. Phys. Lett. 106, 094101 (2015).

[18] K. Smolenski, I. Bazarov, B. Dunham, H. Li, Y. Li, X. Liu, D. Ouzounov, C. Sinclair, D. G. Crabb, Y. Prok et al.,
Design and performance of the cornell ERL DC photoemission gun, AIP Conf. Proc. 1149, 1077 (2009).

[19] J. Maxson, H. Lee, A. C. Bartnik, J. Kiefer, and I. Bazarov, Adaptive electron beam shaping using a photoemission gun and spatial light modulator, Phys. Rev. ST Accel. Beams 18, 023401 (2015).

[20] T. Rublack, J. Good, M. Khojoyan, M. Krasilnikov, F. Stephan, A. Andrianov, E. Gacheva, E. Khazanov, S. Mironov, and E. Syresin, First results attained with the quasi 3-D ellipsoidal photocathode laser pulse system at the high brightness photo injector PITZ, Proc. IPAC (JACoW, Richmond, Virginia, 2015).

[21] C. Hernandez-Garcia, T. Siggins, S. Benson, D. Bullard, H. Dylla, K. Jordan, C. Murray, G. Neil, M. Shinn, and R. Walker, A high average current DC GAAS photocathode gun for ERLS and FELS, in Proceedings of the 21st Particle Accelerator Conference, Knoxville, TN, 2005 (IEEE, Piscataway, NJ, 2005), p. 3117.

[22] T. Rao, A. Burrill, X. Chang, J. Smedley, T. Nishitani, C. H. Garcia, M. Poelker, E. Seddon, F. Hannon, C. Sinclair et al., Photocathodes for the energy recovery linacs, Nucl. Instrum. Methods Phys. Res., Sect. A 557, 124 (2006).

[23] C. Heßler, E. Chevallay, M. Martyanov, V. Fedosseev, I. Martini, and S. Döbert, Recent results on the performance of $\mathrm{Cs} 3 \mathrm{Sb}$ photocathodes in the ring RF-gun, in 6th International Particle Accelerator Conference (IPAC'15), Richmond, VA, USA, 2015 (JACoW, Richmond, Virginia, 2015), p. 1699.

[24] D. Dowell, I. Bazarov, B. Dunham, K. Harkay, C. Hernandez-Garcia, R. Legg, H. Padmore, T. Rao, J. Smedley, and W. Wan, Cathode R\&D for future light sources, Nucl. Instrum. Methods Phys. Res., Sect. A 622, 685 (2010).

[25] M. McIntire, T. Cope, S. Ermon, and D. Ratner, Bayesian optimization of FEL performance at LCLS, in 7th International Particle Accelerator Conference (IPAC'16), Busan, Korea, 2016 (JACOW, Geneva, Switzerland, 2016), p. 2972.

[26] A. Halavanau, G. Ha, G. Qiang, W. Gai, J. Power, P. Piot, E. Wisniewski, D. Edstrom, J. Ruan, and J. Santucci, Microlens array laser transverse shaping technique for photoemission electron source, arXiv:1609.01661.

[27] F. Matsui, S. Goriki, Y. Shimizu, H. Tomizawa, S. Kawato, and T. Kobayashi, Genetic-algorithm-based method to optimize spatial profile utilizing characteristics of electrostatic actuator deformable mirror, Opt. Rev. 15, 156 (2008).

[28] R. Akre, D. Dowell, P. Emma, J. Frisch, S. Gilevich, G. Hays, P. Hering, R. Iverson, C. Limborg-Deprey, H. Loos, A. Miahnahri, J. Schmerge, J. Turner, J. Welch, W. White, and J. Wu, Commissioning the Linac Coherent Light Source injector, Phys. Rev. ST Accel. Beams 11, 030703 (2008).

[29] S. Li, D. Bohler, J. Corbett, A. Fisher, S. Gilevich, Z. Huang, J. Robinson, F. Zhou, R. Fiorito, and E. Montgomery, LCLS injector laser modulation to improve FEL operation efficiency and performance, in Proceedings of IPAC'2015, Richmond, VA, 2015 (JACoW, Richmond, Virginia, 2015), pp. 1813-1815. 
[30] L. Hornbeck, Projection displays and MEMS: timely convergence for a bright future, Proc. SPIE 2639, 2 (1995).

[31] Wavelength Transmittance Considerations for DLP DMD Window, DLPA031C (Texas Instruments, 2012).

[32] S. Lederer, G. Asova, J. Baehr, K. Boyanov, C. Boulware, H. Grabosch, M. Haenel, Y. Ivanisenko, S. Khodyachykh, S. Korepanov et al., Investigations on the thermal emit- tance of Cs2Te photocathodes at PITZ, in Proceedings of FEL07, Novosibirsk, Russia (FEL, 2007).

[33] F. Zhou, A. Brachmann, F. Decker, P. Emma, S. Gilevich, R. Iverson, P. Stefan, and J. Turner, High-brightness electron beam evolution following laser-based cleaning of a photocathode, Phys. Rev. ST Accel. Beams 15, 090703 (2012).

[34] D. Dowell (private communication). 